\title{
The Future of Endoscopic Retrograde Cholangiopancreatography in Korea
}

\author{
Chang Min Cho \\ Department of Internal Medicine, School of Medicine, Kyungpook National University, Daegu, Korea
}

Corresponding Author

Chang Min Cho

ORCID https://orcid.org/0000-0002-9903-1282

E-mail cmcho@knu.ac.kr
See "A National Survey on the Environment and Basic Techniques of Endoscopic Retrograde Cholangiopancreatography in Korea" by Jae Min Lee, et al. on page 904, Vol. 15, No. 6, 2021
Although the advance of noninvasive imaging tests, such as magnetic resonance cholangiopancreatography and endoscopic ultrasonography, has replaced the diagnostic role of endoscopic retrograde cholangiopancreatography (ERCP), ERCP is still an important procedure in the nonsurgical management of various pancreatobiliary diseases. During the past four decades since the first introduction in 1968, tremendous progress has been made in the equipment and technology of ERCP from fiberoptic endoscopes to high-resolution video duodenoscopes with additional devices. While ERCP performance is progressively transforming to a platform for more complex advanced techniques, such as cholangioscopy and ERCP in altered anatomy, ERCP is still one of the most technically demanding endoscopic procedures, with thorough training and a steep learning curve in both technical endoscopic skills and judgement/interpretation and relatively high adverse event rates, ranging from $3 \%$ to $15 \%{ }^{1,2}$

Meanwhile a literature demonstrated that the number of ERCP have decreased since 1996 with steady state until 2000s, according to the national survey of the ERCP practice based on the database of Health Insurance Review and Assessment Service (HIRA), the number of ERCP has been consistently increased in Korea; the annual ERCP rate per 100,000 inhabitants was from 78.6 in 2013 to 91.3 in 2017, which is comparable to 92.5 of the United States in 2013. ${ }^{3,4}$ In particular, the proportion of patients aged 80 and older was increased from $14.3 \%$ in 2012 to $17.2 \%$ in $2015 .{ }^{5}$

In this issue of Gut and Liver, Lee et al. ${ }^{6}$ revealed the national survey on the operational environment and basic technical trends of ERCP in Korea. The study was orga- nized by the committee of policy and quality management in Korean Pancreatobiliary Association (KPBA), where 84 KPBA members participated by completing a predesigned structured questionnaire that contained three categories: (1) the current environment of ERCP; (2) the preparation for ERCP; (3) the current trend of basic techniques for ERCP in Korea.

While $40 \%$ of endoscopists perform fewer than 50 sphincterotomies in Canada and a large number of ERCPs tends to be performed at low volume centers in the United States and the United Kingdom, majority of ERCPs were performed by small number of well-experienced endoscopists in Korea, which is likely attributable to a tendency to be concentrated on doctors in university hospital or tertiary referral centers. ${ }^{5,7,8}$

This survey revealed several variabilities with regard to the work environment and basic techniques of ERCP in Korea comparing with the developed countries. In Western countries, deep sedation or general endotracheal anesthesia and use of nonsteroidal anti-inflammatory drugs (NSAIDs) are preferred methods of sedation and prophylaxis of post-ERCP pancreatitis, respectively. ${ }^{9,10}$ In Korea, ERCP is usually performed under sedation with propofol and/or midazolam by an endoscopist. Due to commercially no availability of rectal NSAIDs, aggressive hydration with lactated Ringer's solution and prophylactic pancreatic stent placement are the preferred preventive management of pancreatitis. There were no significant differences in favorable devices or techniques comparing to other countries.

Whatever the limitations of this study, the current survey provides a representative picture of the ERCP en- 
vironment in Korea. However, unfortunately, due to poor response rate from less experienced endoscopists, these are underrepresented in this survey.

The issue that needs to be overcome in the future is the verification and training of professional power. ERCP is not an occasional job and its practice requires adequate training, credentials, and sufficient volume of cases to maintain expertise.

Based on the comprehensive survey of ERCP practice using the database of HIRA, most ERCP endoscopists were inadequately trained during their periods of fellowship; the number of ERCP performed was less than 150 cases in two-thirds of respondents, and a third of respondents reported that they have been trained to perform ERCP with less than 30 cases. ${ }^{4}$ Although the number of institutions in which ERCP can be performed is consistently increasing in Korea, the number of institutions in which satisfactory ERCP training can be provided is not sufficient yet. Furthermore, the period of ERCP training is too short for fellows to gain adequate experience of ERCP in real clinical practice. Although the problem of large numbers of ERCP trainees performing relatively few ERCP procedures is not unique to Korea, an awareness of this problem should be increased and consideration to improve ERCP training should be made in future. While certified system for upper and lower gastrointestinal endoscopy has been implemented since 2006 in Korea, an established system for certification of ERCP does not exist, which requires longer training period than general endoscopy. Recently, much has been reported about the need to measure and improve the quality of endoscopy services, but still the variability exists in standards used by hospitals for credentialing physicians to ERCP in Korea. There is an urgent need to settle the credentialing process to enhance practice and to protect patients, which suits our society.

In conclusion, ERCP in Korea has come a long way, but there is still much to do in the future such as the quality control of endoscopists. On a national basis, the accepted standards of practice in ERCP are needed to make an outline to ensure consistent clinical standards in patient management.

\section{CONFLICTS OF INTEREST}

C.M.C. is an editorial board member of the journal but was not involved in the peer reviewer selection, evaluation, or decision process of this article. No other potential conflicts of interest relevant to this article were reported.

\section{ORCID}

Chang Min Cho https://orcid.org/0000-0002-9903-1282

\section{REFERENCES}

1. Forbes N, Leontiadis GI, Vaska M, et al. Adverse events associated with endoscopic retrograde cholangiopancreatography: protocol for a systematic review and meta-analysis. BMJ Open 2021;11:e053302.

2. Talukdar R, Nageshwar Reddy D. ERCP in developing countries: the way forward. Gastrointest Endosc 2016;84:667-679.

3. Park JM, Kang CD, Lee JC, Hwang JH, Kim J. Recent 5-year trend of endoscopic retrograde cholangiography in Korea using National Health Insurance Review and Assessment Service open data. Gut Liver 2020;14:833-841.

4. Ahn DW, Han JH, Kim HJ, et al. Practice of endoscopic retrograde cholangiopancreatography in Korea: results from a national survey. Korean J Pancreas Biliary Tract 2019;24:2130 .

5. Yoon SB, Kim J, Paik CN, et al. Trends and characteristics of endoscopic retrograde cholangiopancreatography: a nationwide database study in Korea. Korean J Pancreas Biliary Tract 2021;26:186-194.

6. Lee JM, Moon SH, Park SW, et al. A national survey on the environment and basic techniques of endoscopic retrograde cholangiopancreatography in Korea. Gut Liver 2021;15:904911.

7. Allison MC, Ramanaden DN, Fouweather MG, Davis DK, Colin-Jones DG. Provision of ERCP services and training in the United Kingdom. Endoscopy 2000;32:693-699.

8. Hilsden RJ, Romagnuolo J, May GR. Patterns of use of endoscopic retrograde cholangiopancreatography in a Canadian province. Can J Gastroenterol 2004;18:619-624.

9. Buxbaum J, Roth N, Motamedi N, et al. Anesthetist-directed sedation favors success of advanced endoscopic procedures. Am J Gastroenterol 2017;112:290-296.

10. Elmunzer BJ, Waljee AK, Elta GH, Taylor JR, Fehmi SM, Higgins PD. A meta-analysis of rectal NSAIDs in the prevention of post-ERCP pancreatitis. Gut 2008;57:1262-1267. 